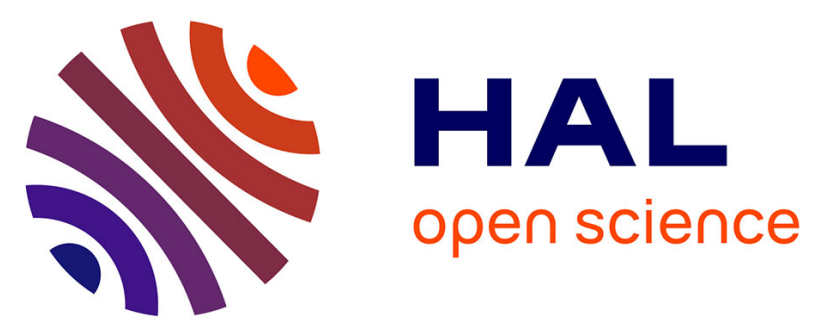

\title{
A PCR-based method for estimating parasitism rates in the olive fly parasitoids Psyttalia concolor and P. lounsburyi (Hymenoptera: Braconidae)
}

Hugo Mathe-Hubert, Jean-Luc Gatti, Marylène Poirie, Thibaut Malausa

\section{- To cite this version:}

Hugo Mathe-Hubert, Jean-Luc Gatti, Marylène Poirie, Thibaut Malausa. A PCR-based method for estimating parasitism rates in the olive fly parasitoids Psyttalia concolor and P. lounsburyi (Hymenoptera: Braconidae). Biological Control, 2013, 67 (1), pp.44-50. 10.1016/j.biocontrol.2013.07.001 . hal-02167849

\author{
HAL Id: hal-02167849 \\ https://hal.science/hal-02167849
}

Submitted on 28 Jun 2019

HAL is a multi-disciplinary open access archive for the deposit and dissemination of scientific research documents, whether they are published or not. The documents may come from teaching and research institutions in France or abroad, or from public or private research centers.
L'archive ouverte pluridisciplinaire HAL, est destinée au dépôt et à la diffusion de documents scientifiques de niveau recherche, publiés ou non, émanant des établissements d'enseignement et de recherche français ou étrangers, des laboratoires publics ou privés. 


\title{
A PCR-based method for estimating parasitism rates in the olive fly parasitoids Psyttalia concolor and P. lounsburyi (Hymenoptera: Braconidae)
}

\author{
Hugo Mathé-Hubert*, Jean-Luc Gatti, Marylène Poirié, Thibaut Malausa \\ Institut National de la Recherche Agronomique (INRA), UMR 1355, Sophia Antipolis, France \\ Centre National de la Recherche Scientifique (CNRS), UMR 7254, Sophia Antipolis, France \\ Université Nice Sophia Antipolis, UFR Sciences, Sophia Antipolis, France
}

\author{
Keywords: \\ Psyttalia spp. \\ Ceratitis capitata \\ Bactrocera oleae \\ Tephritidae \\ Braconidae \\ Cytochrome oxidase I (COI) \\ PCR detection kit
}

\begin{abstract}
Several parasitoids of the genus Psyttalia have been repeatedly introduced as biological control agents against the principal pest of olive, the fly Bactrocera oleae. However, few of the parasitoids released have become established and proved effective against B. oleae. It may however still be possible to find effective biological control agents adapted to local environmental conditions among the highly diverse Psyttalia species and populations infesting $B$. oleae worldwide. For this purpose, we have developed a rapid, sensitive molecular method based on the polymerase chain reaction (PCR) for estimating and comparing the parasitism success of Psyttalia parasitoids through the detection of eggs and larvae within the host. This method was tested and shown to be appropriate for two Psyttalia species (Psyttalia concolor and Psyttalia lounsburyi). The possible detection of DNA was also demonstrated for several populations of these species and for other Psyttalia species, namely Psyttalia humilis and Psyttalia ponerophaga. For P. concolor and $P$. lounsburyi, a strong correlation was observed between the parasitism rates estimated by PCR, host larva dissection and counts of emerging parasitoids. No significant difference was found between the rates of parasitism estimated by host larva dissection and PCR, whereas the rates of parasitism estimated by PCR were significantly higher than those estimated from emergence, suggesting occurrence of mortality during the parasitoid development. This PCR method is thus highly reliable and provides an objective criterion for estimating the efficacy of biological control agent candidates from diverse taxa and populations of Psyttalia.
\end{abstract}




\section{Introduction}

The fruit fly pest Bactrocera oleae (Rossi) (Diptera: Tephritidae) is considered one of the most damaging pests of olive, causing estimated losses of $5 \%$ worldwide and of up to $98 \%$ in some parts of the Mediterranean Basin (Bueno and Jones, 2002). It can rapidly colonize new areas (Zygouridis et al., 2009), and thus occurs in most places in which cultivated or wild olive trees are present. It damages olives in various ways, from oviposition stings on the surface rendering the fruit unsuitable for table consumption, to a loss of production due to fruits rotting and dropping and a decrease in the quality of the oil, which is acidified by the presence of larvae. This damage results in economic losses of approximately US\$ 800 million/year (Bueno and Jones, 2002; Nardi et al., 2005). The main methods for controlling $B$. oleae are mass trapping and insecticide treatments (Daane and Johnson, 2010). However, increasing insecticide resistance in B. oleae (Kakani et al., 2010; Vontas et al., 2001) and the need to decrease pesticide use has led to the repeated testing of biological control, with various degrees of success (Daane and Johnson, 2010; for review, see Daane et al., 2011). The main biological control agents used against $B$. oleae in the last 60 years have been the solitary endoparasitoids of the tephritids Psyttalia concolor, Psyttalia humilis and Psyttalia lounsburyi (Braconidae: Opiinae) (Daane et al., 2011), all of which belong to the P. concolor species complex (Kimani-Njogu et al., 2001; Rugman-Jones et al., 2009). This complex comprises several closely related taxa, most of which are difficult to distinguish morphologically, and some can hybridize (Billah et al., 2007). A strong genetic differentiation has been observed between geographically distant populations of part of these taxa (Cheyppe-Buchmann et al., 2011; Karam et al., 2008; Rugman-Jones et al., 2009), and local adaptation of some taxa to the host or to environmental conditions may occur (Rugman-Jones et al., 2009). Thanks to this diversity, it may still be possible to find appropriate biological control agents combining high levels of successful parasitism with adaptation to local environmental conditions in the target area. However, this will require accurate estimates of parasitism rate (PR) and parasitism success (i.e. the rate of success for parasitoid development within the initially parasitized host) for the candidate parasitoid species or populations.

Molecular-based approaches have largely contributed to improve our knowledge of insect pests and biological control auxiliaries. For instance, a large part of the diversity of tephritid pests and of their parasitoids has been characterized (Jenkins et al., 2012). DNA-barcoding and microsatellite genotyping have notably provided insights on the taxonomy and population structure in the Psyttalia genus (Cheyppe-Buchmann et al., 2011; Rugman-Jones et al., 2009). In contrast, the evaluation of PR, an important parameter for biological control, still encounters technical limitations. One easy-to-perform method, referred to hereafter as the "rearing method", is based on the counting of parasitoid adults emerging from hosts previously exposed to parasitism. However, this method provides only an apparent PR because parasitism may end in the death of the parasitoid or of both the host and the parasitoid, making it difficult, if not impossible, to determine whether the host was initially parasitized (Gariepy et al., 2005, 2007; Gariepy, 2007; Greenstone, 2003, 2006; Jones et al., 2005; Ratcliffe et al., 2002). Another method for estimating PR gets around this problem by estimating the proportion of hosts containing parasitoid eggs through the dissection of host larvae (the "dissection method"). This approach is time-consuming and tedious, and it may lead to more or less severe underestimations of PR depending on whether eggs are difficult to find in the host tissue (Agustí et al., 2005). Examples of PCR-based detection of parasitoid species, whether inside the host or inside the fruit, have been reported (Jenkins et al., 2012), and PR estimation techniques based on such detection of parasitoid eggs within the host are among the best alternatives (Gariepy et al., 2007; Greenstone, 2006). They are increasingly used, as they are less time-consuming and often more reliable than the "dissection" and "rearing" methods. They are also generally more sensitive, cheaper, and they require less expertise compared to other molecular methods aimed at detecting parasitoid specific proteins, e.g. enzyme electrophoresis and serological assays using monoclonal antibodies (Stuart and Greenstone, 1997; for a complete overview, see Greenstone, 2006). We present here a PCR-based method for the detection of eggs of Psyttalia spp. within host larvae, and demonstrate that this method accurately estimates PR from the first day of oviposition, for both $P$. concolor and $P$. lounsburyi. Successful PCR amplification suggests that it may also be appropriate for $P$. humilis and other related taxa. This method will then be useful for estimating the PR of some of the candidate biological control agents of the genus Psyttalia, providing an objective criterion of choice for the various taxa and populations. Moreover, it could also be used for quality control on mass-reared biological control agent populations.

\section{Materials and methods}

\subsection{Biological material}

The $P$. concolor and $P$. lounsburyi populations used to estimate PRs originate from Sicily, Italy (collection in 2010) and Stellenbosch, South Africa (collection in 2005; Cheyppe-Buchmann et al., 2011), respectively. Since collection, they have been reared in the laboratory as described in Thaon et al. (2009), under controlled conditions $\left(22 \pm 1{ }^{\circ} \mathrm{C}\right.$; relative humidity 55\%; photoperiod 16L:8D), on the alternative host Ceratitis capitata. Briefly, a nutrient media (104 g of brewer's yeast, $112 \mathrm{~g}$ of carrot powder, $180 \mathrm{~g}$ of potato flakes, $1.8 \mathrm{~g}$ of Nipagin (Specialites Chimiques Distribution, Gellainville, France), $1.8 \mathrm{~g}$ of sodium Benzoate, $40 \mathrm{~mL}$ of hydrochloric acid at $16,5 \%$ and $900 \mathrm{~mL}$ of water) containing one week-old $C$. capitata larvae is used to coat a ping pong ball, which is then wrapped in stretched Parafilm ${ }^{\mathrm{TM}}$ (Pechiney Plastic Packaging, Chicago, US) and suspended in a cage containing 400 Psyttalia parasitoids to allow parasitism for approximately seven hours. Parasitoid-exposed $C$. capitata larvae are then transferred to a rearing box containing the same nutrient media until parasitoid adults emerge. A more detailed version of the rearing protocol can be found in Benvenuto et al. (2012).

\subsection{Design of $P C R$ primers}

For PCR amplification purposes, sequences from the mitochondrial cytochrome oxidase subunit I gene (COI - LCO/HCO) and from the ribosomal region between the $5.8 \mathrm{~S}$ and $28 \mathrm{~S}$ (including the internal transcribed spacer 2 [ITS2]) were tested. Primers were designed so as to ensure amplification of the target sequence from Psyttalia species ( $P$. concolor and $P$. lounsburyi), while avoiding amplification from the host species ( $B$. oleae and $C$. capitata). The available sequences for the host and parasitoid species (six ITS2 and two COI sequences for the hosts; eight ITS2 and 34 COI sequences for the parasitoids) were recovered from GenBank (ITS2 sequences: EU761063 and EU761064, EU761048 to EU761052, AF276515, AF276516, AY209010, AF332590, AF187102, AF189691, AF307848 and DQ490237; COI sequences: EU761020 to EU761025, GU725008 to GU725031, EU761036 to EU761038, DQ116368 and GQ505009) and aligned with the Clustal W program (Larkin et al., 2007). Primers were designed with Primer 3 
software (Rozen and Skaletsky, 2000). Six and four primer pairs were chosen for amplification of the COI and ITS2 sequences, respectively. Following PCR, gel electrophoresis and sequencing of some of the PCR products, we found that only two pairs of primers for ITS2 and one for COI successfully amplified the expected fragments in the various conditions tested. However, the ITS2 primers gave less reliable and sensitive results than the COI primers. We therefore retained the COI primer pair (Forward; 5'-GTTTATTAATAAATGATCAGATTTATAATAG-3'; Reverse: 5'AAAATTGCTAAATCAACTGAAG-3'), which amplifies a 307 bp fragment, for the PCR test.

\subsection{DNA extraction and amplification}

We extracted DNA from parasitized or unparasitized C. capitata second-instar larvae or from parasitoid adults as follows. Individual specimens were placed in each well of a 96-well plate (Fullskirt PCR Plates AB-2800, Thermo-Fisher Scientific, Brumath, France) with $20 \mu \mathrm{L}$ of DNA extraction solution (prepGEM ${ }^{\mathrm{TM}}$ Insect kit, ZyGEM, Hamilton, New Zealand). DNA extraction was then performed according to the prepGEM ${ }^{\mathrm{TM}}$ Insect kit protocol, with a modified enzymatic digestion procedure $\left(2 \mathrm{~h}\right.$ at $75^{\circ} \mathrm{C}$ rather than $15 \mathrm{~min}$ ). For host larvae, two steps have been added to the extraction protocol: before the enzymatic digestion, individual were crushed by adding one $2 \mathrm{~mm}$-diameter glass marble per well, sealing the plates with flat-cap strips (AB-0784, Thermo-Fisher) and shaking the plates with a motorized Mixer Mills MM 301 (Retsch $\mathrm{GmbH}$, Haan, Germany) until each larva was crushed. After the enzymatic digestion, the plate was centrifuged for $15 \mathrm{~min}$ at $2000 \mathrm{~g}$ and $2 \mu \mathrm{L}$ out of the $20 \mu \mathrm{L}$ of supernatant were used as DNA template for PCR.

PCR was carried out with the QIAGEN Multiplex PCR Kit (Qiagen, Hilden, Germany), in a total volume of $15 \mu \mathrm{L}$. The selected forward and reverse primers were each used at a final concentration of $0.4 \mu \mathrm{M}$. PCR conditions were $95^{\circ} \mathrm{C}$ for $15 \mathrm{~min}, 40$ cycles of $94^{\circ} \mathrm{C}$ for $30 \mathrm{~s}, 52{ }^{\circ} \mathrm{C}$ for $60 \mathrm{~s}$ and $72{ }^{\circ} \mathrm{C}$ for $60 \mathrm{~s}$, and a final extension at $72^{\circ} \mathrm{C}$ for $10 \mathrm{~min}$. PCR-amplified fragments were analyzed with the QIAxcel Advanced System and the QIAxcel DNA Fast Analysis Kit (QIAGEN).

\subsection{Assessment of PCR sensitivity}

We assessed the sensitivity of the PCR method as follows: a pool of six P. concolor eggs (obtained by the dissection of parasitoid females) was submitted to the DNA extraction procedure in $80 \mu \mathrm{L}$ of extraction solution. Amounts of DNA equivalent to 0.7, $0.4,0.2$, and 0.1 eggs were then used independently to spike the extract of one non parasitized C. capitata larva in a final volume of $20 \mu \mathrm{L}$. PCR was then carried out, as described above, with $1 / 10$ th of the volume of each spiked DNA extract (final amounts of $P$. concolor eggs DNA equivalent to $0.07,0.04,0.02$ and 0.01 eggs). The dilution and PCR steps were repeated three times.

\subsection{Assessment of PCR specificity}

The PCR specificity was first tested in silico by comparing the alignment of the mitochondrial $\mathrm{COI}$ sequences used to design the primers (EU761020 to EU761025, GU725008 to GU725031 and EU761036 to EU761038) to the COI sequences of P. humilis, Psyttalia phaeostigma, Psyttalia cosyrae, Psyttalia perproxima, and Psyttalia ponerophaga (respectively: EU761026 to EU761031; EU761045; EU761039 to EU761042; EU761032 to EU761035 and EU761043; EU761015 to EU761019). Unfortunately, there was no COI sequence available for Psyttalia dacicida. We then assessed the specificity of the primers in situ by performing individual PCR assays on adult parasitoids from different populations and species belonging to the Psyttalia genus. To this end, $32 \mathrm{P}$. concolor (Corsica, collection in 2012, $N=5$; Crete, collection in 2010, $N=10$; Sardinia, collection in 2012, $N=17$ ), 6 P. lounsburyi (Kenyan strain reared in the laboratory since 2003), $5 P$. ponerophaga (Pakistani strain reared at the EBCL, Montpellier, France) and 8 P. humilis (Otavi, Namibia, collection in 2008) were used. Nine negative controls were added to test for occurrence of false positives due to DNA contamination. The identity of the amplified products was confirmed by sequencing.

\subsection{Comparison of the three detection methods in P. concolor and validation on P. lounsburyi}

We evaluated the $P$. concolor PR on $C$. capitata larvae by three methods (see introduction part): the "rearing method", the "dissection method" and the "PCR method". Six host groups (ping-pong balls of $5 \mathrm{~cm}$ diameter containing a large number of second-instar host larvae) were exposed to parasitism in a same box containing approximately 1-3 weeks-old Psyttalia (200 males and $200 \mathrm{fe}-$ males), but with three different length of exposure: $1 \mathrm{~h}$ (low expected PR), $5 \mathrm{~h}$ (medium expected PR) and $8 \mathrm{~h}$ (high expected PR). Following parasitism, each host group was divided into 5 subgroups of unequal size, in which the PR was estimated by one of the three methods. Two subgroups (15-42 larvae) were used for the PCR, and two subgroups (13-38 larvae) for the dissection method, the analysis being performed either $8 \mathrm{~h}$ ("day 0"; first subgroup) or one day ("day 1"; second subgroup) after the beginning of exposure to Psyttalia. The fifth subgroup, composed of all remaining larvae (several hundred) was used to estimate the apparent PR by the "rearing" method (ratio of the number of adult parasitoids to the total number of Ceratitis pupae estimated after the completion of development of the remaining larvae). The number of larvae used to estimate the PR by the three methods, for each group, each exposure length and each development period post-exposure is indicated in Table 1. For the PCR method, we systematically added at

Table 1

Parasitism rate (PR) estimated for the various subgroups of host larvae with different PR estimation methods.

\begin{tabular}{|c|c|c|c|c|c|c|c|}
\hline \multirow[t]{2}{*}{ Species } & \multirow{2}{*}{$\begin{array}{l}\text { Parasitism } \\
\text { duration }\end{array}$} & \multirow[t]{2}{*}{ Group } & \multicolumn{2}{|l|}{ PCR } & \multicolumn{2}{|l|}{ Dissection } & \multirow[t]{2}{*}{ Rearing } \\
\hline & & & Day 0 & Day 1 & Day 0 & Day 1 & \\
\hline \multirow[t]{6}{*}{ P. concolor } & $1 \mathrm{~h}$ & 1 & $0.10(N=20)$ & $0.00(N=22)$ & $0.10(N=20)$ & $0.11(N=18)$ & $0.04(N=523)$ \\
\hline & & 2 & $0.29(N=28)$ & $0.14(N=22)$ & $0.30(N=27)$ & $0.12(N=33)$ & $0.06(N=677)$ \\
\hline & $5 \mathrm{~h}$ & 3 & $0.27(N=15)$ & $0.18(N=22)$ & $0.31(N=13)$ & $0.25(N=20)$ & $0.20(N=635)$ \\
\hline & & 4 & $0.66(N=29)$ & $0.66(N=15)$ & $0.42(N=31)$ & $0.37(N=24)$ & $0.30(N=359)$ \\
\hline & $8 \mathrm{~h}$ & 5 & $0.36(N=14)$ & $0.50(N=42)$ & $0.63(N=19)$ & $0.71(N=24)$ & $0.37(N=611)$ \\
\hline & & 6 & $0.73(N=15)$ & $0.33(N=21)$ & $0.53(N=38)$ & $0.48(N=25)$ & $0.31(N=413)$ \\
\hline P. lounsburyi & $8 \mathrm{~h}$ & & $0.61(N=99)$ & $0.19(N=32)$ & $0.47(N=55)$ & $0.35(N=20)$ & $0.31(N=75)$ \\
\hline
\end{tabular}

Values in brackets are the number of individuals (larvae or pupae for the rearing method) used to estimate PR. 
least 10 negative controls per PCR plate to check for occurrence of false positives due to DNA contamination.

For validation of the method on P. lounsburyi, PR was estimated by PCR and dissection at "day 0 " and "day 1 " on one group of $C$. capitata larvae exposed to parasitism for $8 \mathrm{~h}$, as described above.

\subsection{Statistical analysis}

The three methods used to estimate PR for $P$. concolor were compared with "parasitized vs unparasitized" binary data, with a binomial GLMM (glmer function of the lme4 package; Bates et al., 2010). The method (PCR at "day 0", PCR at "day 1", dissection at "day 0 ", dissection at "day 1 ", rearing method) was considered as a fixed effect. The experimental replicate (groups subjected to the same treatment) was considered as a random effect. Post-hoc comparisons were then performed with the "multcomp" package (Hothorn et al., 2008). We also checked for overdispersion of the data with the following formula: the Pearson goodness-of-fit statistic divided by the residual degree of freedom (McCullagh and Nelder, 1989). Finally, Spearman's rank correlation coefficients were calculated to assess the agreement between the methods. The mean PR for each length of exposure to parasitism were weighted to give the same weight to all methods and larval groups exposed to parasitism. For $P$. lounsburyi, we used the same method as for $P$. concolor, except that no random effect was considered in the model since data were collected only for one length of exposure to parasitism. We thus used a classical GLM ("glm" function of "R"), and, for the same reason, no correlation coefficient was calculated.

\section{Results}

\subsection{Development and validation of the PCR method}

To develop a PCR method suitable for estimating the parasitism rate in Psyttalia species, we designed primers predicted to amplify either part of the COI or the ITS2 sequence in P. concolor and P. lounsburyi but not in the hosts B. oleae and C. capitata. As results with ITS2 primers proved less reliable and sensitive, we focused on the use of COI primers for validation of the parasitoid egg detection method in $P$. concolor and $P$. lounsburyi. COI amplification was specific to Psyttalia DNA, as demonstrated by the production of a single
307 bp fragment when $P$. concolor or $P$. lounsburyi DNA was present in the PCR solution, versus the absence of PCR products when only DNA from B. oleae or C. capitata was present (Fig. 1A). The PCR method described also proved highly sensitive since it detected an amount of Psyttalia egg DNA as low as that of $1 / 100$ of an individual egg (Fig. 1B). This ensures that eggs could be detected in parasitized samples even if the extraction of parasitoid DNA was of low efficiency.

\subsection{Comparison of the three detection methods in P. concolor}

For $P$. concolor, the mean PR obtained with the various methods increased with the length of exposure to parasitism: $12 \% \pm 9.7$ after $1 \mathrm{~h}, 36 \% \pm 17.4$ after $5 \mathrm{~h}$, and $52 \% \pm 15$ after $8 \mathrm{~h}$ (values calculated from raw data in Table 1 weighted to give the same weight to all methods and larval groups; mean PR \pm standard deviation).

This made it possible to assess the covariation of the methods (using data from PCR and dissection at times "day 0" and "day 1 ", and from the rearing method) with different PR values. Estimates obtained with the different methods at different times were roughly similar with some notable exceptions; the PR estimates obtained by PCR and dissection were not significantly different $(P=0.57$ at "day 0"; $P=0.84$ at "day 1 "), but those obtained with the rearing method were highly significantly lower than those obtained with the two other methods at "day 0 " $(P<0.001$ with dissection; $P<0.001$ with $P C R)$ and significantly lower than those obtained by dissection at "day 1 " $(P=0.02$ with dissection, $P=0.32$ for PCR; see Fig. 2). With the PCR method, estimated PR was higher at "day 0" than at "day 1 " $(P=0.015$; Fig. 2) while the trend toward higher PR values at day 0 than at day 1 was not significant for the dissection method $(P=0.93$; Fig. 2$)$.

High Spearman's correlation coefficients were obtained between PR estimates obtained by the PCR and dissection methods $(r=0.77, P=0.10$ at "day 0"; $r=0.83, P=0.058$ at "day 1 " and $r=0.81, P<0.001$ for estimates based on pooled "day 0 " and “day 1").

\subsection{Validation on P. lounsburyi}

All the trends observed with $P$. concolor were also observed with $P$. lounsburyi. The mean PR estimate with the various methods was $38 \% \pm 16$ (as for $P$. concolor, the mean was weighted so as to give
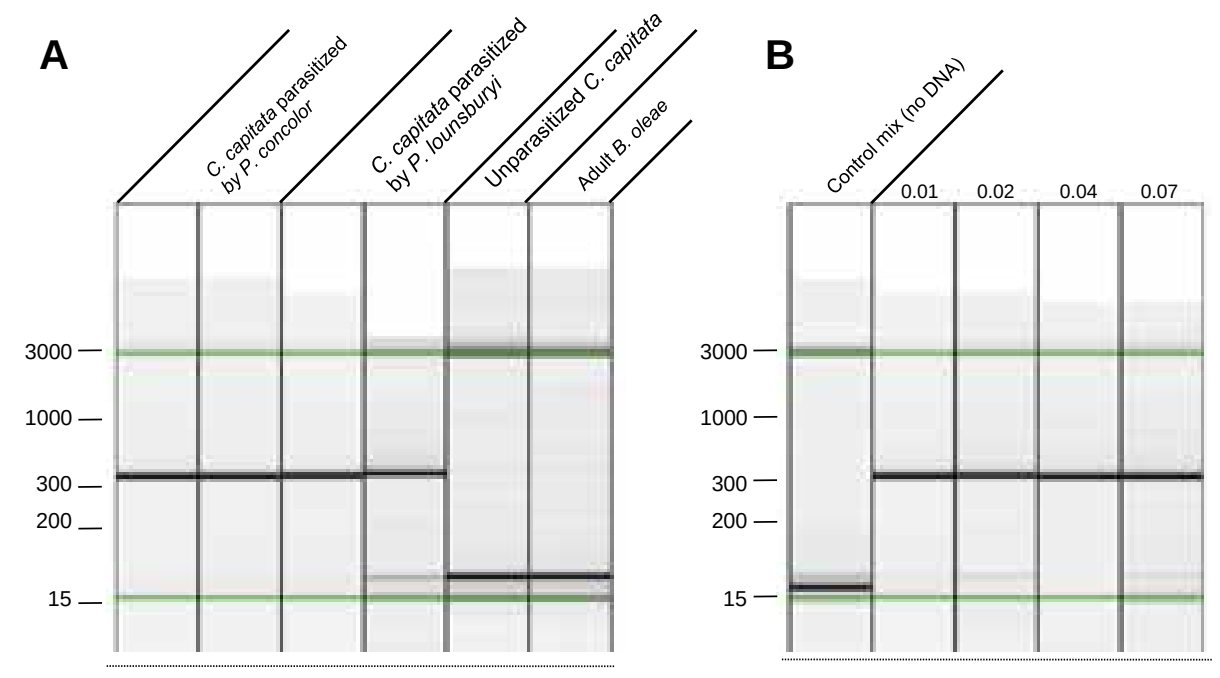

Fig. 1. PCR results, as generated with the Qiaxcel Advanced System. Each lane shows the electrophoresis result for a single sample. Fig. 1A Illustrates specificity of the primers for Psyttalia species. Fig. 1B shows the high sensitivity of the PCR method (the lanes correspond to one of the three replicates from the sensitivity experiment). The values indicated above the lanes are the number of eggs used to spike the DNA. Fragment size estimates were obtained with the Qiagen 15 bp-3 kb alignment marker (Cat no: 929522), corresponding to the bands under the green lines. They are displayed in bp on the left. (For interpretation of the references to colour in this figure legend, the reader is referred to the web version of this article.) 


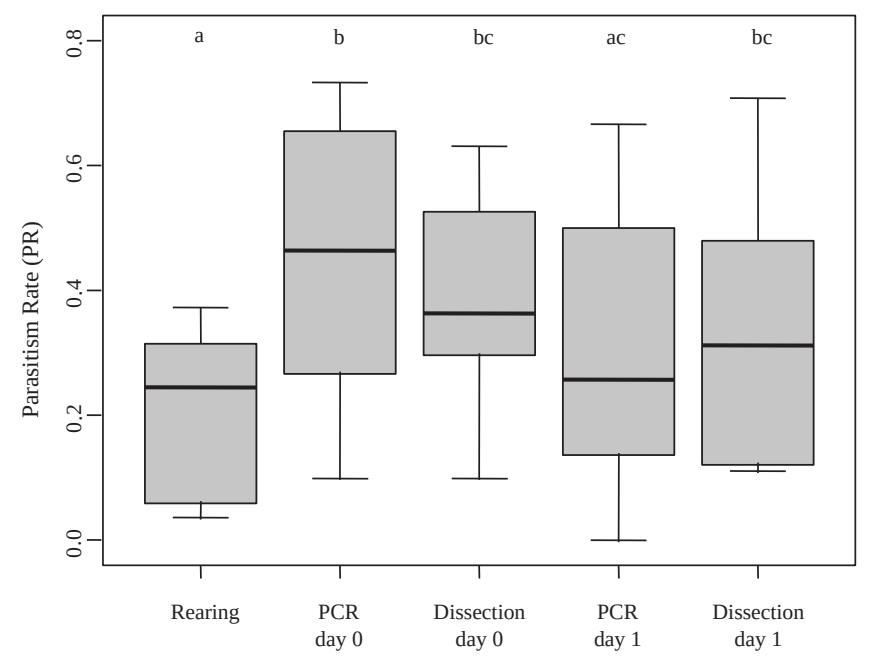

Fig. 2. Observed parasitism rates for $P$. concolor estimated by the different methods. Methods are the rearing method, PCR at "day 0", dissection at "day 0", PCR at "day 1 ", dissection at "day 1". The box plots represent the PR estimates obtained for the various groups of larvae exposed to $P$. concolor oviposition for different periods of time. The letters indicate the level of significance for multiple comparison tests.

the same weight to each method). The PR estimates obtained by PCR and dissection were not significantly different $(P=0.49$ with the group "day 0"; $P=0.68$ with the group "day 1 ") while the estimates obtained with the "rearing" method and with the PCR method at "day 1" were significantly lower than those obtained by the PCR method at "day 0" $(P<0.001$ in both cases). Thus, as for $P$. concolor, PCR yielded a higher estimate of PR at "day 0" than at "day 1 ". With the dissection method, a similar trend was observed but the PR estimated at "day 0" was not significantly higher than that estimated at "day 1 " ( $P=0.87$; Fig. 3$)$.

Among the nine PCR plates used to measure PR by $P$. concolor and $P$. lounsburyi, only one of the 93 negative controls provide a positive result. We nevertheless decided to retain the corresponding plate for the analysis since the contamination rate $(10 \%$ for the plate, $1.08 \%$ over all plates) remained low and did not affect the overall results.

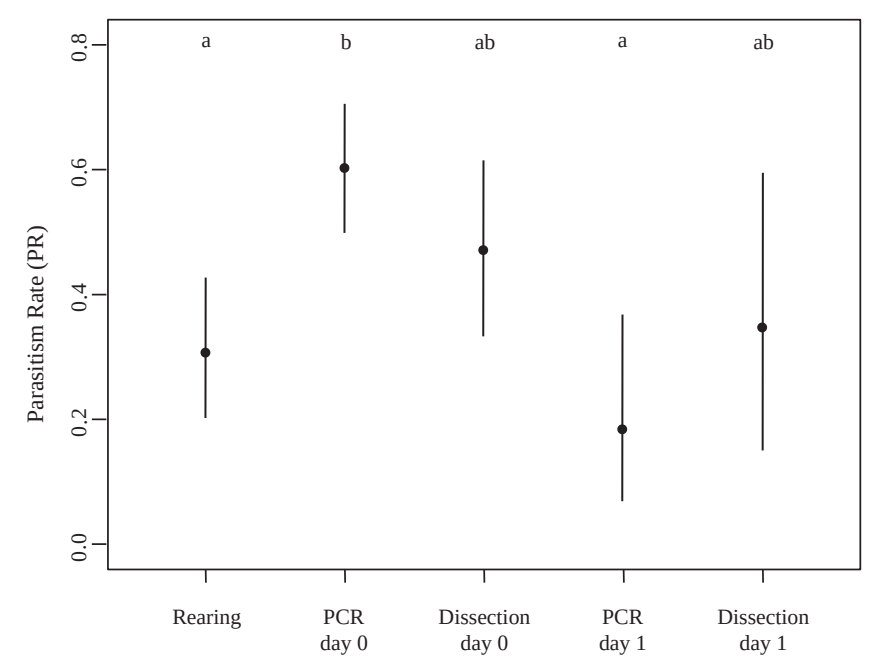

Fig. 3. Observed parasitism rates for $P$. lounsburyi estimated by the differen methods. Methods are the rearing method, PCR at "day 0", dissection at "day 0", PCR at "day 1", dissection at "day 1". Dots indicate the PR estimated for the group of larvae exposed to $P$. lounsburyi oviposition for $8 \mathrm{~h}$. Error bars indicate the $95 \%$ confidence interval. Letters indicate the level of significance in multiple comparison tests.

\subsection{Assessment of PCR specificity}

In silico analyses revealed that the COI primers we designed were exactly matching to $P$. lounsburyi, $P$. concolor and $P$. humilis sequences only. Differences at one or two positions were observed for each primer when compared to the sequences of $P$. phaeostigma, $P$. cosyrae or $P$. ponerophaga. For $P$. perproxima, only the reverse primer differed at two positions.

PCR amplification assays were then performed on individuals from three populations of $P$. concolor $(N=32$ in total) and one population of $P$. lounsburyi $(N=6)$ other than those used to develop the parasitism-detection method, as well as on individuals from one population of the species $P$. humilis $(N=8)$ and $P$. ponerophaga $(N=5)$. Successful amplifications were obtained for all tested Psyttalia individuals (data not shown).

\section{Discussion}

\section{1. $P C R$ is an effective method for estimating early $P R$}

We have developed a reliable and sensitive method for the early detection of parasitism by $P$. concolor and $P$. lounsburyi. This method was able to detect parasitoid DNA down to levels corresponding to $1 / 100$ of an egg, and the PR estimated by PCR was not significantly different from that obtained by the dissection of host larvae. Furthermore, the correlation between PR estimates by PCR and dissection was highly significant regardless of the time at which PR was estimated ( 8 or $24 \mathrm{~h}$ post-parasitism). This confirms the value of molecular methods for such applications (Agustí et al., 2005; Gariepy et al., 2007; Greenstone, 2006). Moreover, this PCR method can be used to detect eggs at very early stages of parasitoid development, which should provide a better estimate of PR because of the occurrence of parasitoid mortality (Gariepy, 2007; Gariepy et al., 2005; Jones et al., 2005; Ratcliffe et al., 2002; for reviews, see: Gariepy et al., 2007; Greenstone 2003, 2006). Indeed, we observed that PR estimates at early stages of parasitoid development (via PCR or dissection) were higher than those obtained after the completion of host and parasitoid development (rearing method). The comparison of mean PRs estimated just after oviposition and at the end of the development (rearing method) suggests mortality rates of about $46 \%$ and $43 \%$ for P. concolor and P. lounsburyi, respectively. These high values are nevertheless within the range of those usually reported in other parasitoid species (Gariepy, 2007; Gariepy et al., 2005; Jones et al., 2005; Ratcliffe et al., 2002). Interestingly, PR estimates by PCR just after oviposition were also higher than those obtained $24 \mathrm{~h}$ later, an observation that is in agreement with the much higher difference between the apparent PR (rearing method) and the PCR-based estimates at "day 0", compared to "day 1 ". This suggests that Psyttalia parasitoids death mainly occurs during the first $24 \mathrm{~h}$ after oviposition, and that the PCR method is sensitive enough not to be significantly influenced by the egg development. The small size of the recently-laid egg is indeed generally thought to result in the underestimation of PCR-based PR at early stages of development (Gariepy, 2007; Gariepy et al., 2005; Jones et al., 2005; Weathersbee et al., 2004). The parasitoid death in the first $24 \mathrm{~h}$ may be explained by the oviposition of non viable eggs and/or by the host immune reaction. We never observed encapsulated eggs in C. capitata but for instance in Drosophila paramelanica, dead endoparasitoid eggs can be observed as early as 6$12 \mathrm{~h}$ post-infection in absence of any encapsulation reaction (Carton et al., 2009). If the difference between PR estimates at the two time points can indeed be explained by egg mortality, it should be observed whatever the method used (PCR or dissection) while significant differences occurred only with the PCR method. However, this may result from the difficulty in finding eggs by dissection a 
few hours after oviposition while the PCR method may be sensitive enough to detect even traces of DNA from eggs in the process of degradation.

\subsection{Applications of the developed PCR method}

PCR detection methods are often designed for field studies, for instance, the monitoring of a released biological control agent (e.g. Jenkins et al., 2012). To this end, PCR primers are generally highly specific to one particular parasitoid species or population (the parasitoid to be monitored). Our aim here was to develop a unique tool for the comparison of several populations and species within the Psyttalia genus. We thus designed primers from a region that tends to be more conserved than other regions at the genus level. PCR assays demonstrated successful amplification of the target sequence from the four $P$. concolor and the two $P$. lounsburyi populations tested, as well as from the $P$. humilis and $P$. ponerophaga species. In silico assays suggest that this PCR method can also be used with $P$. phaeostigma, $P$. cosyrae and $P$. perproxima and may possibly be appropriate for several other parasitoid species of the Psyttalia genus. Indeed, the number of differences between the primers sequence and the target sequence is not higher for $P$. phaeostigma, $P$. cosyrae and $P$. perproxima than for $P$. ponerophaga.

Comparing the PR of different species through PCR with a single pair of primers should make comparisons more reliable when evaluating several candidate biological control agents. However, this lack of specificity may lead to a misinterpretation of results in field studies if not combined with an accurate taxonomic characterization of sampled individuals. If necessary, this PCR method may be followed by a RFLP or sequencing analysis. Indeed, complementary analyses are easy to perform here because the amplified fragment is part of the COI region used for DNA barcoding (LCO/HCO).

The PCR method, combined with the rearing method, will also allow comparison of the parasitism success in different populations and closely related taxa. Parasitism can lead to three alternatives outcomes (i) the death of both the host and the parasitoid, (ii) the host recovery and (iii) the emergence of the parasitoid, which probability can be estimated from parallel estimations of early PR by PCR and apparent PR (rearing method). Indeed, the parasitism success can be calculated as the ratio of the apparent PR to the PR at day 0 while a "host basal mortality rate" can be estimated using the rearing method on a group of unparasitized hosts of known size. The comparison of this "basal mortality rate" with the observed mortality rate of a group of hosts of known PR allows to calculate the "mortality rate of parasitized larvae". Finally, the comparison of the "parasitism success rate" and the "mortality rate of parasitized larvae" allows to deduce the rate of host recovery.

Detailed comparisons of parasitism success would be relevant in many biological control programs. Indeed, geographic variations of the outcome of host-parasitoid interactions are frequently observed. A biological control agent that is effective in one area may then be maladapted in another, which could be detected by a parasitism success analysis. This analysis would also be useful for the quality control of laboratory strains. Indeed, laboratory conditions (e.g. the use of a non natural host or an artificial diet) may lead to the evolution of unwanted features (Chailleux et al., 2012; Hoffmann et al., 2001; Hopper and Roush, 1993; Hufbauer, 2002; Tayeh et al., 2012). Parasitism success and PR may not necessarily provide the same information since they do not respond similarly to environmental conditions. For example, PR is thought to be strongly influenced by behavioural factors and, thus, highly changeable, whereas parasitism success is thought to be more strongly influenced by physiological factors and then less affected by environmental variation (Canale and Benelli, 2011).

\section{Conclusion}

In conclusion, we describe here a rapid and accurate method for detecting early parasitism by P. lounsburyi and P. concolor based on the use of a single protocol. This should make it possible to assess the variability in parasitism success at the intra- and interspecific levels, and thus to guide the choice of candidate biological control agents from the genus Psyttalia in programs for the biological control of the olive fruit fly.

\section{Acknowledgments}

We thank Marcel Thaon, Nicolas Ris and Fabrice Pustoc'h for maintaining the populations of Ceratitis flies and Psyttalia parasitoids, and for their help in the design of the PCR experiment, as well as Dominique Colinet for fruitful discussions and Marie Claude Bon from EBCL (Montpellier, France) for great help in supplying Psyttalia samples. We also thank the three anonymous reviewers for their comments that largely helped improving the manuscript. This work was funded by the French "Bibliothèque du Vivant" project and the INRA Department "Santé des Plantes et Environnement". H. Mathé-Hubert is supported by a Ph.D. funding from the "Provence Alpes Côte d'Azur" (PACA) French region and the INRA“Santé des Plantes et Environnement" Department.

\section{References}

Agustí, N., Bourguet, D., Spataro, T., Delos, M., Eychenne, N., Folcher, L., Arditi, R., 2005. Detection, identification and geographical distribution of European corn borer larval parasitoids using molecular markers. Mol. Ecol. 14, 3267-3274.

Bates, D., Maechler, M., Bolker, B., 2010. lme4: Linear mixed-effects models using S4 classes. R package version 0.999375-39.

Benvenuto, C., Cheyppe-Buchmann, S., Bermond, G., Ris, N., Fauvergue, X., 2012. Intraspecific hybridization, life history strategies and potential invasion success in a parasitoid wasp. Evol. Ecol. 26, 1311-1329.

Billah, M.K., Kimani-Njogu, S.W., Wharton, R.A., Overholt, W.A., Wilson, D.D., Cobblah, M.A., 2007. Cross mating studies among five fruit fly parasitoid populations: potential biological control implications for tephritid pests. BioControl 53, 709-724.

Bueno, A.M., Jones, O., 2002. Alternative methods for controlling the olive fly, Bactrocera oleae, involving semiochemicals. IOBC WPRS Bull. 25, 147-156.

Canale, A., Benelli, G., 2011. Impact of mass-rearing on the host seeking behaviour and parasitism by the fruit fly parasitoid Psyttalia concolor (Szépligeti) (Hymenoptera: Braconidae). J. Pest Sci. 85, 65-74.

Carton, Y., Frey, F., Nappi, A.J., 2009. Parasite-induced changes in nitric oxide levels in Drosophila paramelanica. J. Parasitol. 5, 1134-1141.

Chailleux, A., Desneux, N., Seguret, J., Do Thi Khanh, H., Maignet, P., Tabone, E., 2012. Assessing European Egg Parasitoids as a Mean of Controlling the Invasive South American Tomato Pinworm Tuta absoluta. PLoS One 7, 1-8.

Cheyppe-Buchmann, S., Bon, M.-C., Warot, S., Jones, W., Malausa, T., Fauvergue, X., Ris, N., 2011. Molecular characterization of Psyttalia lounsburyi, a candidate biocontrol agent of the olive fruit fly, and its Wolbachia symbionts as a pre-requisite for future intraspecific hybridization. Biocontrol 56, 713-724.

Daane, K.M., Johnson, M., Pickett, C., Sime, K., Wang, X.G., Nadel, H., Andrews, J.W., Hoelmer, K., 2011. Biological controls investigated to aid management of olive fruit fly in California. Calif. Agric. 65, 21-28.

Daane, K.M., Johnson, M.W., 2010. Olive fruit fly: managing an ancient pest in modern times. Annu. Rev. Entomol. 55, 151-169.

Gariepy, T.D., 2007. Molecular Markers for Lygus Parasitoids to Assess Host Specificity of Candidate Entomophagous Biological Control Agents.

Gariepy, T.D., Kuhlmann, U., Gillott, C., Erlandson, M., 2007. Parasitoids, predators and PCR: the use of diagnostic molecular markers in biological control of Arthropods. J. Appl. Entomol. 131, 225-240.

Gariepy, T.D., Kuhlmann, U., Haye, T., Gillott, C., Erlandson, M., 2005. A single-step multiplex PCR assay for the detection of European Peristenus spp., parasitoids of Lygus spp. Biocontrol Sci. Technol. 15, 481-495.

Greenstone, M.H., 2003. Assessing insect endoparasitism by PCR: applications to classical biological control, in: Proceedings of the 1st International Symposium on Biological Control of Arthropods. pp. 98-101.

Greenstone, M.H., 2006. Molecular methods for assessing insect parasitism. Bull. Entomol. Res. 96, 1-13.

Hoffmann, M.P., Ode, P.R., Walker, D.L., Gardner, J., Van Nouhuys, S., Shelton, A.M., 2001. Performance of Trichogramma ostriniae (Hymenoptera: Trichogrammatidae) reared on factitious hosts, including the target host, Ostrinia nubilalis (Lepidoptera: Crambidae). Biol. Control 21, 1-10.

Hopper, K.R., Roush, R.T., Powell, W., 1993. Management of genetics of biologicalcontrol introductions. Annu Rev Entomol 38, 27-51. 
Hothorn, T., Bretz, F., Westfall, P., 2008. Simultaneous inference in general parametric models. Biom. J. 50, 346-363.

Hufbauer, R.A., 2002. Evidence for nonadaptive evolution in parasitoid virulence following a biological control introduction. Ecol. Appl. 12, 66-78.

Jenkins, C., Chapman, T.A., Micallef, J.L., Reynolds, O.L., 2012. Molecular techniques for the detection and differentiation of host and parasitoid species and the implications for fruit fly management. Insects 3, 763-788.

Jones, D.B., Giles, K.L., Chen, Y., Shufran, K.A., 2005. Estimation of hymenopteran parasitism in cereal aphids by using molecular markers. J. Econ. Entomol. 98 217-221.

Kakani, E.G., Zygouridis, N.E., Tsoumani, K.T., Seraphides, N., Zalom, F.G., Mathiopoulos, K.D., 2010. Spinosad resistance development in wild olive fruit fly Bactrocera oleae (Diptera: Tephritidae) populations in California. Pest Manag. Sci. 66, 447-453.

Karam, N., Guglielmino, C.R., Bertin, S., Gomulski, L.M., Bonomi, A., Baldacchino, F., Simeone, V., Malacrida, A.R., 2008. RAPD analysis in the parasitoid wasp Psyttalia concolor reveals Mediterranean population structure and provides SCAR markers. Biol. Control 47, 22-27.

Kimani-Njogu, S., Trostle, M.K., Wharton, R.A., Woolley, J.B., Raspi, A., 2001 Biosystematics of the Psyttalia concolor species complex (Hymenoptera: Braconidae: Opiinae): the identity of populations attacking Ceratitis capitata (Diptera: Tephritidae) in coffee in Kenya. Biol. Control 20, 167-174.

Larkin, M.A., Blackshields, G., Brown, N.P., Chenna, R., McGettigan, P.A., McWilliam, H., Valentin, F., Wallace, I.M., Wilm, A., Lopez, R., Thompson, J.D., Gibson, T.J. Higgins, D.G., 2007. Clustal W and Clustal X version 2.0. Bioinformatics 23, 2947-2948.

McCullagh, P., Nelder, J.A., 1989. Generalized Linear Models, . Monographs on Statistics and Applied Probability, Second Edition. Chapman \& Hall/CRC Lavoisier.fr.

Nardi, F., Carapelli, A., Dallai, R., Roderick, G.K., Frati, F., 2005. Population structure and colonization history of the olive fly, Bactrocera oleae (Diptera, Tephritidae) Mol. Ecol. 14, 2729-2738.
Ratcliffe, S.T., Robertson, H.M., Jones, C.J., Bollero, G.A., Weinzierl, R.A., 2002. Assessment of parasitism of house fly and stable fly (Diptera: Muscidae) pupae by pteromalid (Hymenoptera: Pteromalidae) parasitoids using a polymerase chain reaction assay. J. Med. Entomol. 39, 52-60.

Rozen, S., Skaletsky, H., 2000. Primer3 on the WWW for general users and for biologist programmers. Methods Mol. Biol. 132, 365-386.

Rugman-Jones, P.F., Wharton, R., Noort, T.V., Stouthamer, R., 2009. Molecular differentiation of the Psyttalia concolor (Szépligeti) species complex (Hymenoptera: Braconidae) associated with olive fly, Bactrocera oleae (Rossi) (Diptera: Tephritidae), in Africa. Biol. Control 49, 17-26.

Stuart, M.K., Greenstone, M.H., 1997. Immunological Detection of Hymenopteran Parasitism in Helicoverpa zea and Heliothis virescens. Biol. Control 202, 197202.

Tayeh, A., Estoup, A., Laugier, G., Loiseau, A., Turgeon, J., Toepfer, S., Facon, B., 2012. Evolution in biocontrol strains: insight from the harlequin ladybird Harmonia axyridis. Evol. Appl. 5, 481-488.

Thaon, M., Blanchet, A., Ris, N., 2009. Contribution à l'optimisation de l'élevage du parasitoïde. Cahier Technique de l'INRA 66, 21-31.

Vontas, J.G., Cosmidis, N., Loukas, M., Tsakas, S., Hejazi, M.J., Ayoutanti, A. Hemingway, J., 2001. Altered Acetylcholinesterase confers organophosphate resistance in the olive fruit fly Bactrocera oleae. Pest. Biochem. Physiol. 71, 124132.

Weathersbee, A.A., Shufran, K.A., Panchal, T.D., Dang, P.M., Evans, G.A., 2004 Detection and differentiation of parasitoids (Hymenoptera: Aphidiidae and Aphelinidae) of the brown citrus aphid (Homoptera: Aphididae): speciesspecific polymerase chain reaction amplification of $18 \mathrm{~S}$ rDNA. Ann. Entomol. Soc. Am. 97, 286-292.

Zygouridis, N.E., Augustinos, A.A., Zalom, F.G., Mathiopoulos, K.D., 2009. Analysis of olive fly invasion in California based on microsatellite markers. Heredity 102 402-412. 